\title{
Postsuburbia ou posturbia ? Les mégaprojets résidentiels dans la périphérie de Santiago du Chili
}

Post-suburbia or post-urbia? The residential megaprojects in the periphery of Santiago de Chile

Post-suburbia oder Post-urbia? Die Großwohnsiedlungen in der Peripherie von Santiago-de-Chile

\section{Rodrigo Hidalgo D}

\section{OpenEdition}

Journals

Édition électronique

URL : http://journals.openedition.org/rge/340

DOI : $10.4000 /$ rge.340

ISSN : 2108-6478

\section{Éditeur}

Association des géographes de l'Est

Édition imprimée

Date de publication : 1 juin 2005

Pagination : 209-217

ISSN : 0035-3213

Référence électronique

Rodrigo Hidalgo D, «Postsuburbia ou posturbia ? Les mégaprojets résidentiels dans la périphérie de Santiago du Chili », Revue Géographique de l'Est [En ligne], vol. 45 / 3-4 | 2005, mis en ligne le 10 juin 2009, consulté le 08 septembre 2020. URL : http://journals.openedition.org/rge/340 ; DOI : https:// doi.org/10.4000/rge.340

Ce document a été généré automatiquement le 8 septembre 2020.

Tous droits réservés 


\title{
Postsuburbia ou posturbia? Les mégaprojets résidentiels dans la périphérie de Santiago du Chili ${ }^{1}$
}

\author{
Post-suburbia or post-urbia? The residential megaprojects in the periphery of \\ Santiago de Chile \\ Post-suburbia oder Post-urbia? Die Großwohnsiedlungen in der Peripherie von \\ Santiago-de-Chile
}

Rodrigo Hidalgo D

\section{NOTE DE L'ÉDITEUR}

Article reçu le 22 août 2005, accepté le 12 décembre 2005

Adaptation : Michel Deshaies

L'auteur remercie vivement le Dr. Axel Borsdorf pour sa collaboration dans la genèse de ce texte, ainsi que le professeur Alejandro Salazar pour la révision du texte en français (traduction libre) ${ }^{2}$.

1 Les transformations des différentes composantes de la morphologie urbaine peuvent servir de révélateurs pour comprendre les changements qui se produisent dans les villes. Dans les métropoles latino-américaines, les aires centrales et leurs périphéries montrent d'importantes ruptures dans leur trame routière et dans leurs espaces fonctionnels. Cela peut comporter la construction d'autoroutes, qui couvrent des espaces sans cesse plus importants, ainsi que la modification constante de l'espace interne, caractérisée par une augmentation remarquable de la hauteur des constructions, mais aussi par l'expansion périphérique, conséquence des utilisations à dominante résidentielle (Borsdorf, et Hidalgo, 2004a).

2 Ces espaces résidentiels expriment tous une dualité extrême, avec d'une part des logements sociaux produits par l'État, et d'autre part, des espaces fermés ou des quartiers habités non seulement par les élites, mais aussi par les classes moyennes et 
moyennes inférieures, qui sont présentes sur des espaces dont la composition sociale est ainsi différente (Borsdorf, 2000 ; Thuillier, 2000 ; Meyer et Bähr, 2001 ; Kohler, 2002 ; Kanitscheider, 2002 ; Lacarrieu et Thuillier 2004 ; Hidalgo et Borsdorf, 2005).

3 Les quartiers fermés, conçus comme des espaces de logements à l'accès contrôlé, ayant aussi des dispositifs de surveillance et de sécurité, sont de nouveaux éléments qui marquent l'organisation de l'espace urbain et qui modèlent différemment la physionomie actuelle de la ville latino-américaine (Borsdorf, Bähr et Janoschka 2002 ; Mertins, 2003). En ce qui concerne les causes de ce processus, certains auteurs comme Borsdorf (2002) et Borsdorf et Hidalgo (2004a) soulignent la proximité de la culture ibérique ; d'autres, tels que De Mattos (2003) ou Parnreiter, Fisher et Jäger (2005) en rendent responsables la mondialisation. Cependant, vu les tendances récentes, un consensus pourrait être dégagé, car les mégaprojets résidentiels apparus depuis un certain temps rompent avec toute la tradition urbaine en Amérique latine. Ainsi, ceuxci peuvent être considérés comme des conséquences de la mondialisation, avec des expressions particulières pour chacun des pays où ils sont apparus.

Ces mégaprojets sont des espaces résidentiels concentrant de 500000 à plus de 100000 habitants. Il s'agit d'un nouveau concept de ville entourée de murs, de clôtures, de grandes barrières et de systèmes de sécurité pour protéger leurs habitants, en les isolant du monde extérieur. Ce sont des communautés pourvues de leurs propres prestations de services, équipements et approvisionnements (autrement dit, autosuffisantes) ainsi que réservées uniquement à une certaine couche sociale. De temps en temps - comme dans le cas de Santiago du Chili - ces mégaprojets se raccordent à la ville-centre par des autoroutes privées, assez souvent uniquement utilisées par les habitants qui demeurent dans ces complexes résidentiels (Borsdorf et Hidalgo, 2005).

5 Des phénomènes semblables avaient déjà été analysés à São Paulo (Coy et Pöhler ; 2002 : Alphaville) et ont été aussi l'objet d'une étude détaillée de Janoschka (2002) sur la ville de Buenos Aires (Nordelta). Contrairement au cas de São Paulo, les effets possibles de ce type d'intervention à Santiago, où plusieurs mégaprojets éparpillés dans la périphérie de l'agglomération urbaine sont déjà partiellement en cours, restent toujours méconnus (voir Hidalgo, 2004).

6 Cet article vise à analyser le phénomène des "villes clôturées » à Santiago du Chili (Hidalgo, 2004) et à constater s'il s'agit bien d'un processus "pseudo-urbain» qui comporte des tendances de "post-urbanisation", si bien que ces interventions pourraient être considérées comme l'antithèse du concept de ville. Les exemples du Brésil et de l'Argentine sont seulement utilisés à titre de comparaison; les idées exposées ici se fondent sur des travaux de terrain effectués pendant les années 2004 et 2005 à Santiago. Le socle empirique du travail correspond à un cadastre des ensembles de logements prévus pour une population de plus de 50000 habitants, actuellement en construction au nord de l'Aire Métropolitaine de Santiago (AMS $\left.{ }^{3}\right)$, plus spécifiquement, dans les communes de Lampa et de Colina, Province de Chacabuco (figure 1). L'interprétation et la proposition théorique du processus de construction des nouvelles périphéries résidentielles clôturées dans l'AMS a été faite à partir de la confection de cartes thématiques, d'entretiens et d'une base de données géoréférencée des projets résidentiels (voir et comparer avec Borsdorf et Hildago 2004b et Borsdorf et Hildago, 2005). 
Figure 1 : Pourcentage de variations entre recensements de la population au niveau communal dans l'AMS, 1992-2002.

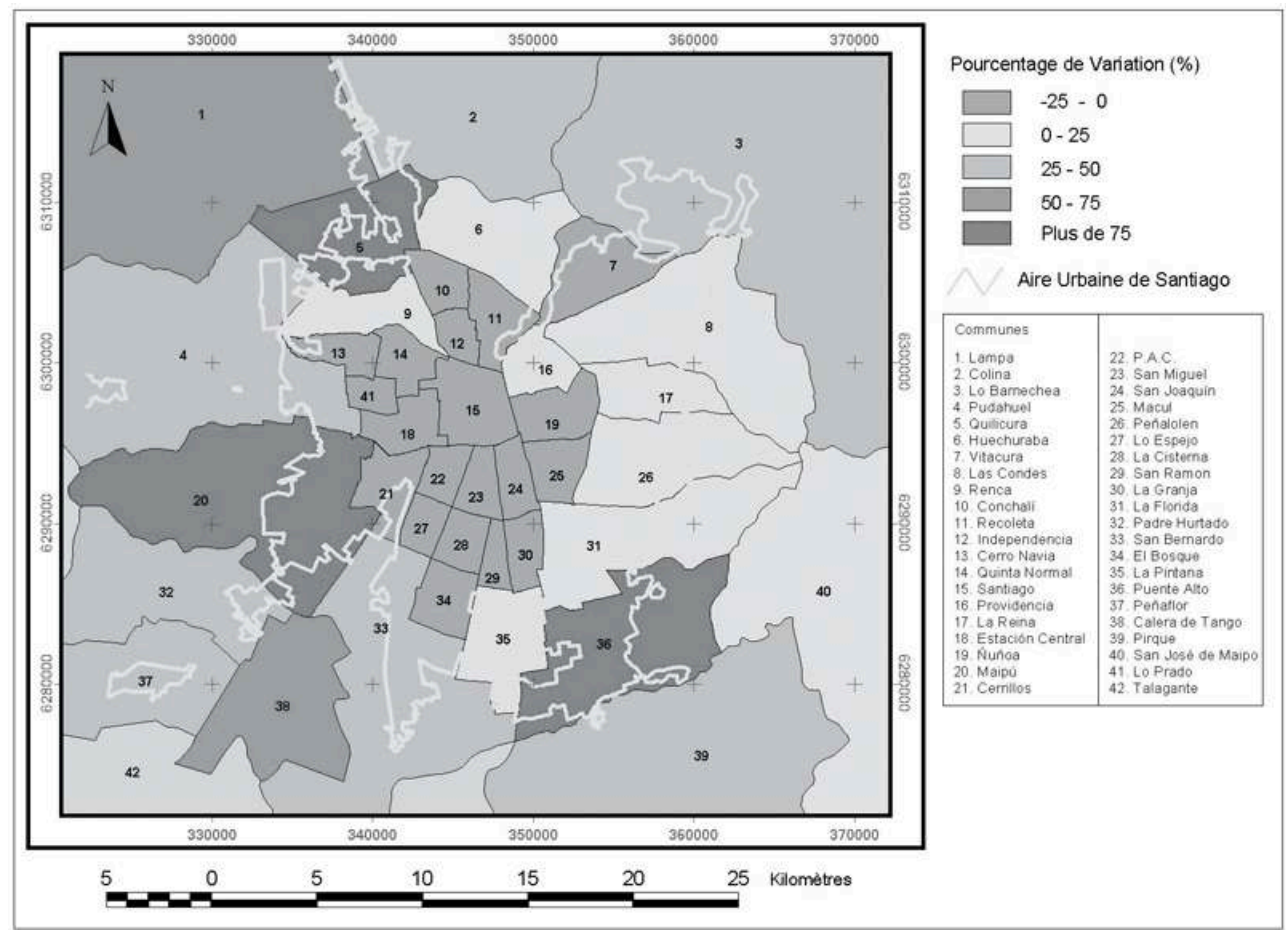

\section{L'expansion métropolitaine et les nouvelles périphéries résidentielles en Amérique latine. Enjeux pour la ville de Santiago}

7 Si l'on prend en considération le schéma signalé par Harvey (1990), dans lequel la mondialisation est mise en rapport avec une série d'innovations dans les technologies de l'information, de la communication et du transport, entrânant aussi le dérèglement croissant de l'économie et de l'activité du secteur public, on assiste en même temps, comme un moteur de la mondialisation, à la diffusion d'un nouveau paradigme économique à caractère capitaliste. Ce paradigme, dénommé néo-libéralisme préconise la libéralisation des frontières tarifaires, le recul de l'investissement public, l'assouplissement du marché du travail et l'exaltation des avantages comparatifs entre les différentes régions de la planète (Sassen, 1994, Dollfus, 1997).

Cette rétroaction entre la mondialisation et le néo-libéralisme a une expression territoriale évidente dans les villes où elle a pris naissance: en l'occurrence, le dérèglement du marché du sol et la spéculation immobilière (Harvey, 1990 ; Marcuse et Kempen, 2000). Ainsi, pendant les deux dernières décennies, les villes ont expérimenté de nouvelles formes d'expansion, en passant d'une structure relativement compacte, pour arriver à une distribution dispersée des espaces périphériques des villes. Cela fait penser aux archipels urbains, ressemblant à la réalité observée dans une partie des cas européens ou anglo-américains (Monclús, 1998 ; De Matteis, 1998). 
Dans quelques pays du nord de l'Europe, l'expansion urbaine associée à la diffusion de la mondialisation et du néo-libéralisme, a été mise en rapport avec le surgissement d'un continuum ville-campagne, lié à la structuration d'une "ville régionale », tout au long des principales voies de communication. Plus récemment, cela a été lié à l'avènement d'un "post-suburbain», se différenciant des formes précédentes (les banlieues) par une plus grande prospérité et par une structure de flux plus complexe que la simple relation fonctionnelle des flux quotidiens entre l'espace suburbain et la ville (Borsdorf 2004a, 2004b).

10 En Amérique latine, les périphéries métropolitaines commencent aussi à montrer des mutations dans leurs formes d'expansion, en présentant une diversité d'utilisations marquée par la fragmentation: des autoroutes urbaines et suburbaines, des quartiers fermés et de grands centres commerciaux (shopping centers ou malls), font partie de la triade qui donne lieu à un nouveau modèle d'organisation métropolitaine. Il s'agit d'un modèle plus fragmenté et polycentrique que la ville héritée du processus de développement fondé sur l'industrialisation induite par l'État (ville bipolaire), et d'une conséquence indirecte de l'application de politiques de développement néo-libérales, fondatrices du « royaume » de la mondialisation (Borsdorf et Hidalgo, 2005).

11 Concernant les périphéries résidentielles, la transition entre la ville polarisée et la ville fragmentée fait référence au passage d'une organisation urbaine caractérisée par un espace social à deux pôles clairement différenciés, "riches » et "pauvres ", vers une organisation dans laquelle ces deux groupes sociaux commencent à se disperser au bord des métropoles, très souvent de manière concentrée (Hidalgo et Borsdorf, 2005). Ainsi, la fragmentation met l'accent sur la complexité des changements actuels de la ville, l'idée centrale étant que la seule condition sociale ne détermine plus la localisation géographique. Elle met aussi en évidence le fait que la société en archipel produit une imbrication des espaces différenciés et accorde une plus grande visibilité aux contrastes (Prêvot, 2000). Bien qu'elle semble être un paradoxe, la fragmentation permet d'établir un lien entre les dynamiques spatiales liées à la métropolisation (l'expansion physique, la mobilité, le surgissement de nouvelles centralités) et l'éclatement de l'unité, résultat de l'amplification des inégalités sociales, des formes de désolidarisation et des regroupements par affinité sociale (Prêvot, 2002).

12 La mondialisation, le néo-libéralisme, les nouveaux espaces suburbains et la fragmentation urbaine constituent le cadre général des nouvelles périphéries résidentielles associées aux mégaprojets des quartiers fermés, qui commencent à être observés à Santiago du Chili dès le début du XXI ${ }^{\mathrm{e}}$ siècle. L'étape suivante est donc l'explication des particularités de ce phénomène pour le cas chilien: ses agents promoteurs, ses conséquences sur l'organisation de l'espace métropolitain, ses projections futures et les changements que les "villes clôturées" sont en train de provoquer sur les formes d'exclusion résidentielle.

\section{Les mégaprojets résidentiels dans la périphérie de Santiago du Chili : le cadre juridique}

Depuis quelques décennies, la croissance de Santiago du Chili (1992-2002: 8,6\%), tout comme celle d'autres métropoles latino-américaines (Borsdorf 2004), reste en dessous du taux moyen du pays (1992-2002: 12,8\%). Toutefois, la ville continue à montrer un 
accroissement de sa population et une augmentation de sa surface, processus différentiel pour l'ensemble des communes qui composent son agglomération. Cette croissance se concentre particulièrement dans les communes périphériques, lesquelles parviennent presque à doubler leur population, par rapport à 1992 (figure 1).

L'espace résidentiel de ces communes est composé essentiellement de quartiers fermés et de logements sociaux, sous la forme de projets résidentiels de tailles diverses, habités par des couches sociales différentes. D'importants mouvements migratoires entre les communes péricentrales et ces ensembles résidentiels sont à l'origine d'une nouvelle configuration territoriale des périphéries. Bien que les habitants aient tendance à migrer vers des territoires de niveau socio-économique équivalent à celui de leur lieu d'origine, des modifications commencent à apparaître. C'est ainsi que d'importants contingents de la classe moyenne migrent vers des communes à niveau socioéconomique moins favorable et chaque fois plus éloignées du noyau dur des communes urbaines de l'AMS (Ortiz et Morales, 2002).

Dans ce contexte, en relation avec le développement de l' immobilier, des processus spéculatifs autour des sols ruraux commencent à apparaitre vers le milieu des années 1980 dans les communes périurbaines du nord de l'agglomération (notamment celles de Colina et Lampa). Ceci a amenéde grands acheteurs fonciers à y investir avec l'objectif d'effectuer une plus value à moyen et à long terme (environ 5 à 20 années), à partir des terres acquises au prix du sol agricole.

Dans un premier temps, en raison de la situation périphérique des terrains situés dans la vallée de Chicureo (commune de Colina) et de leur absence de connexion au réseau routier les reliant à la ville-centre, le développement de ces affaires immobilières est passé inaperçu. Toutefois, la distance à vol d'oiseau entre ces espaces situés dans la vallée de La Dehesa (commune de Lo Barnechea) et les zones plus aisées de Santiago, est de presque dix kilomètres. C'est pour cette raison que les entreprises immobilières ont réalisé avec l'aide de l'État, la construction d'autoroutes privées pour relier ces terrains à l'agglomération. Ainsi, les slogans publicitaires soulignaient au début leur proximité avec les zones à hauts revenus, facteur promotionnel d'une augmentation des prix de vente des terrains.

17 En même temps, les investisseurs se sont mobilisés pour obtenir une modification des outils d'aménagement urbain, objectif qu'ils parviennent à matérialiser vers la fin des années 1990, par la création d'un cadre techno-juridique ayant seulement une application dans le cadre de l'approbation du Plan Régulateur Intercommunal de la Province de Chacabuco de 1997 ( PRICH $^{4}$ ), au nord de l'AMS. Dans ce cadre, des « zones de développement urbain conditionné » (ZODUC), considérées comme des territoires aménageables (de plus de 300 hectares) sont alors apparues et comportent des normes minimales d'équipement et de services avec une densité de construction des logements à respecter. Les ZODUC, conçues pour les groupes sociaux à moyens et moyens-hauts revenus, ont été définies comme des lieux exclusivement résidentiels, seuls $5 \%$ de leur surface étant réservés à des projets éventuels de logements sociaux.

Le PRICH règle le développement urbain des trois communes qui composent cette province: Colina, Lampa et Til-Til, où jusqu'à son approbation, s'est développé un intense processus de fractionnement des sols ruraux en "parcelles d'agrément». Celles-ci sont nées après l'application du «Décret avec Force de Loi » (DFL) 3516 de 1980 , qui a établi dans son article $1^{\text {er }}$ que « les propriétés rurales, c'est-à-dire les biens à usage agricole, d'élevage ou sylvicole, localisées hors des limites urbaines ou des limites 
signalées par les Plans régulateurs intercommunaux de Santiago, Valparaíso et Concepción, pourront être librement divisées par leurs propriétaires, pourvu que les lots résultants aient une surface minimale de 0,5 hectares » (Ministère de l'Agriculture, 1980).

Jusqu'à l'approbation du PRICH, on a enregistré dans ces communes, en application de ce décret, environ 80000 subdivisions de terres (Colina 42 247, Lampa 22253 et Til-Til 15 554) pour une surface totale d'environ 130000 hectares. Ce chiffre double la surface déjà urbanisée de l'AMS pour l'année 2002. Bien qu'il ne soit pas possible d'occuper toutes ces terres à des fins résidentielles, ces chiffres montrent l'ampleur de la subdivision du sol rural en "parcelles d'agrément", ainsi que l'intérêt des propriétaires fonciers pour de futures spéculations.

La dispersion du peuplement dans les espaces périurbains induite par les «parcelles d'agrément", a provoqué une nette détérioration de l'environnement en raison du déficit sanitaire (Hidalgo, Salazar, Roa et Lazcano, 2003) résultant de l'insuffisance des équipements et des infrastructures, ainsi que de l'absence de réseaux d'assainissement (notamment d'eau potable et d'égouts). Ces éléments ont fait partie des arguments utilisés, tant par les chefs d'entreprise que par les fonctionnaires de l'État, pour justifier la mise en place des « ZODUC ", permettant l'augmentation des basses densités de construction dans les « parcelles d'agrément » (Poduje et Yañez, 2000).

21 En parallèle aux ZODUC, le PRICH a mis en place une autre forme juridique d'aménagement dénommée "Aire de Développement Urbain Prioritaire » (AUDP). Ces aires, localisées principalement dans la commune de Lampa, ont été spécialement conçues comme un outil légal permettant l'accueil des projets de logements unifamiliaux destinés à la classe moyenne et moyenne inférieure. Ainsi, les AUDP doivent permettre d'accueillir une partie de la population de la province et dans une plus large mesure des citadins provenant de la grande conurbation de Santiago, en consacrant $30 \%$ de leur surface aux ensembles de logements sociaux.

\section{Les ZODUC actuellement : des « non villes » pour les élites}

Dans la commune de Colina existent actuellement trois ZODUC en voie de construction, à savoir : Piedra Roja, Valle Norte et La Reserva, localisées dans la vallée de Chicureo. Piedra Roja, prévue pour 65000 habitants, est à présent la plus grande, avec la population la plus nombreuse, et une grande quantité d'équipements et de services. La zone de Valle Norte, adjacente à Piedra Roja, devrait concentrer près de 57000 habitants et celle de $L a$ Reserva, 12000 (tableau 1). Bien que cette dernière zone ne puisse pas être considérée comme un mégaprojet, du point de vue du poids démographique (plus de 50000 habitants), elle constitue l'un des projets les plus ambitieux et les plus «selects». Des architectes de renommée mondiale ont réalisé les maquettes des villas de standing qui s'implanteront sur de grands terrains paysagés de manière spectaculaire. Conçu sur le principe des quartiers fermés, l'accès demeure réservé aux résidents ou aux visiteurs. Ces ensembles résidentiels constituent l'avant-garde du développement de l'AMS vers le nord du bassin de la ville de Santiago. 
Tableau 1 : Caractéristiques des principaux (méga) projets résidentiels clôturés dans la Province de Chacabuco, Santiago du Chili.

\begin{tabular}{|c|c|c|c|c|c|}
\hline Nom du projet & Piedra Roja & Valle Norte & La Reserva & $\begin{array}{l}\text { Hacienda } \\
\text { Urbana } \\
\text { Larapinta }\end{array}$ & Valle Grande \\
\hline Modalité & ZODUC & ZODUC & ZODUC & AUDP & AUDP \\
\hline Immobilière & $\begin{array}{l}\text { Immobilaria } \\
\text { Manquehue }\end{array}$ & $\begin{array}{l}\text { Fernandez } \\
\text { Wood, ECSA, } \\
\text { Almagro S.A. }\end{array}$ & $\begin{array}{l}\text { Empresas } \\
\text { Harseim }\end{array}$ & SOCOVESA & Novaterra \\
\hline Localisation & Chicureo & Chicureo & Chicureo & $\begin{array}{l}\mathrm{km} \mathrm{14,} \\
\text { Camino Lo } \\
\text { Echevers- } \\
\text { Lampa }\end{array}$ & $\begin{array}{l}\text { Av. La } \\
\text { Montaña, } \\
\text { Ruta } 5 \text { Norte }\end{array}$ \\
\hline Commune & Colina & Colina & Colina & Lampa & Lampa \\
\hline $\begin{array}{l}\text { Taille du projet } \\
\text { (en ha) }\end{array}$ & 1300 & 1600 & 740 & 300 & 480 \\
\hline $\begin{array}{l}\text { Nombre de } \\
\text { logements }\end{array}$ & 12000 & $* * *$ & 4500 & 8500 & $12000-15000$ \\
\hline $\begin{array}{lr}\text { Nombre } & \text { total } \\
\text { d'habitants } & d u \\
\text { projet } & \end{array}$ & $62000-65000$ & 57000 & $* * *$ & 50000 & 60000 \\
\hline $\begin{array}{l}\text { Période } \\
\text { construction }\end{array}$ & $2002-2022$ & $* * *$ & $2001-2013$ & 2004-2019 & 2004-2016 \\
\hline Connectivité & Pie Andino & $\begin{array}{l}\text { Pie Andino, } \\
\text { Radial } \\
\text { Nororiente }\end{array}$ & $\begin{array}{l}\text { Pie Andino, } \\
\text { Américo } \\
\text { Vespucio } \\
\text { Norte } \\
\text { Express, } \\
\text { Radial } \\
\text { Nororiente }\end{array}$ & $\begin{array}{l}\text { Ruta } \\
5 \text { Norte, } \\
\text { Vespucio } \\
\text { Norte } \\
\text { Express }\end{array}$ & $\begin{array}{l}\text { Ruta } 5 \text { Norte, } \\
\text { Vespucio } \\
\text { Norte Express }\end{array}$ \\
\hline $\begin{array}{l}\text { Investissement } \\
\text { (Millions US\$) }\end{array}$ & 1850 & 700 & 500 & $* * *$ & 690 \\
\hline
\end{tabular}

Source : Information obtenue sur le terrain, dans les bureaux des entreprises respectives et par la diffusion des projets sur l'Internet

$\star \star \star$ : Sans information

La première étape du projet Piedra Roja est à présent en cours, avec 400 maisons vendues, dont la plupart sont déjà habitées (figure 2). Il est structuré sous la forme de "petits quartiers»; les rues principales sont organisées en avenues de desserte et d'accès vers des voies secondaires, très souvent encombrées. Un grand étang de quatre 
hectares constitue un point d'attraction visuel et fonctionnel. Sur ses bords on trouve un club nautique, un centre commercial en construction et de grandes surfaces réservées aux futures utilisations commerciales. Afin de satisfaire les goûts de la haute société, on a prévu des installations exclusives pour la pratique du golf, de l'équitation et du polo.

Figure 2 : Un regard sur les logements des ZODUC ; Piedra Roja, Valle Norte et La Reserva.

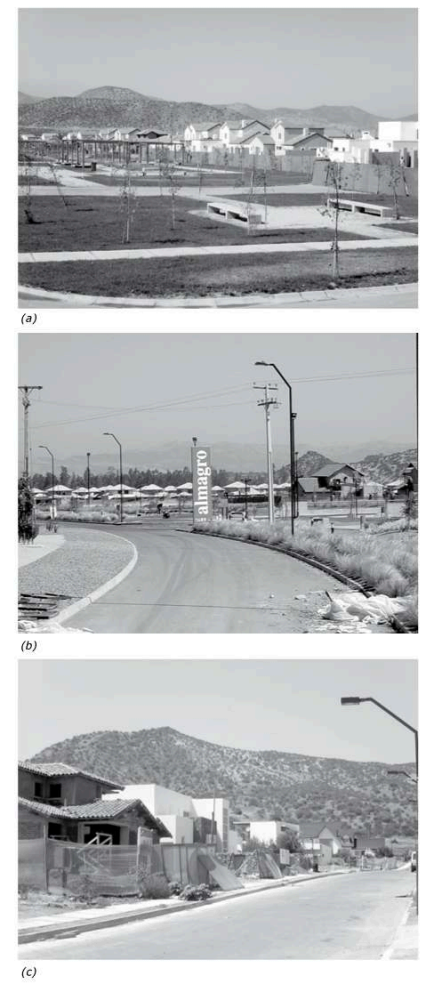

Photographies : (a) Piedra Roja (Axel Borsdorf, janvier 2005) - (b) Valle Norte (Rodrigo Hidalgo, janvier 2005) - (c) La Reserva (Axel Borsdorf, janvier 2005). aux résidents une circulation libre d'encombrement automobile et leur permet d'arriver en quelques minutes à leur travail ou aux établissement scolaires situés dans la vallée de La Dehesa. En prenant l'autoroute General San Martín, on peut rejoindre l'autoroute Vespucio Norte Express et l'autoroute Centrale, des voies express à péage qui permettent de réduire considérablement le temps de trajet.

L'homogénéité architecturale de Piedra Roja, incorporant des principes esthétiques qui rappellent les critères angloamericains plutôt qu'européens, attire l'attention. Les acheteurs peuvent choisir entre cinq types de maisons de plans et de dimensions différentes, mais toujours dans le même style. Ceux qui préfèrent un immeuble plus original, peuvent acheter leur terrain à Hacienda Chicureo (un quartier encore plus exclusif) où l'on bénéficie d'une plus grande liberté de conception architecturale.

Le projet Valle Grande correspond plus ou moins au même modèle de développement : une ville clôturée pour environ 57000 habitants, moins d'infrastructures exclusives, et une architecture plus standardisée orientée vers un segment socio-économique inférieur à celui de Piedra Roja (figure 2). 
27 Par contraste, le projet La Reserva, est destiné aux segments les plus aisés. Situé dans un véritable amphithéâtre montagneux, chaque terrain bénéficie d'un cadre paysager fabuleux. Presque 60 types de maisons à l'architecture futuriste ont été conçus par 40 architectes de renommée internationale (figure 2). La première étape de construction comprend 211 maisons. Au mois de janvier 2005, 160 immeubles étaient déjà vendus et 12 réservés.

28 La route Pie Andino ou Juan Pablo II (à l'origine privée et aujourd'hui en accès libre) est le catalyseur qui stimule les projets urbains à Chicureo. Avec ses 20 mètres de large, trois voies exemptes de tunnels et un faible trafic, elle offre une bonne desserte : à la vitesse de $50 \mathrm{~km} / \mathrm{h}$, on peut parcourir la totalité de la route $(10,5 \mathrm{kms})$ en dix minutes environ. Sa construction, avec la participation de fonds privés et publics, a coûté 15 millions US\$.

Une autre autoroute privée actuellement en construction (Radial Nororiente) permettra la liaison avec les quartiers plus aisés de Santiago. Beaucoup plus onéreuse avec ses 16 $\mathrm{kms}$ de longueur, étant donné les obstacles topographiques qu'elle doit franchir, elle a deux voies dans chaque sens et deux tunnels de 7,6 km de longueur. En roulant à 100 $\mathrm{km} / \mathrm{h}$ de moyenne, on peut parcourir en douze minutes le trajet entre Chicureo et Vitacura. Etant donné la complexité des ouvrages de génie civil, il est prévu que les travaux se termineront en 2007.

30 Une autre route en construction est l'Avenida del Valle, à usage également privé. Cette avenue de seulement $6 \mathrm{kms}$ de long qui parcourt la vallée de Chicureo, relie ces « villes clôturées » avec l'autoroute urbaine de Vespucio Norte Express et ses lignes de service. La construction réalisée pour un investissement de l'ordre de 6,5 millions US\$ se terminera au milieu de l'année 2005.

\section{Les AUDP : de véritables ghettos géants pour les couches moyennes inférieures}

31 Si l'on compare la densité d'habitants de La Réserva (16 hab/ha), de Valle Norte (35 hab/ ha) et de Piedra Roja (50 hab/ha), avec la population prévue dans les AUDP de Larapinta et de Valle Grande (respectivement 167 et $125 \mathrm{hab} / \mathrm{ha}$ ) (tableau 1), il apparaît que la densité démographique dans ces dernières est bien supérieure. Cette situation est révélatrice de la géographie sociale résultant de l'avènement des «villes clôturées ».

Le développement de l'infrastructure routière a aussi favorisé la commune de Lampa, dont la distance avec le centre de l'AMS, en termes de temps de trajet, a diminué grâce à la rénovation de la route 5 Norte et de l'Autoroute Centrale. D'autre part, l'autoroute Vespucio Nord Express a notablement amélioré l'accessibilité de la commune à partir de l'aéroport international. Ceci ouvre la possibilité de déployer dans la commune d'intéressantes offres foncières destinées aux activités industrielles, qui constituent une part considérable des développements sur le territoire communal. Dans le même sens, la «Ciudad Empresarial», pôle d'entreprises de services avec quelques caractéristiques de technopôle, se trouve seulement à $15 \mathrm{~km}$ à vol d'oiseau de la commune de Lampa, et les secteurs industriels et de services associés à cette activité dans la commune de Quilicura, sont aussi situés dans le voisinage de Lampa. Toutefois, cette commune présente des contraintes naturelles. Les secteurs de plaine, libres de constructions, sont des terrains inondables (Castro, 2005). Ces contraintes limitent dans 
l'ensemble l'utilisation agricole des terres et une surface considérable reste par conséquent sans utilisation ou en friche.

Cependant, la proximité de la commune par rapport à l'agglomération centrale et l'amélioration de son accessibilité favorisent le développement de nouveaux parcs industriels, par exemple, Valle Grande, lié actuellement à un mégaprojet résidentiel homonyme.

Les projets de Valle Grande etde Larapinta ciblent les jeunes ménages des couches moyennes et moyennes inférieures. Ils comprennent des logements de 55, 65, 70 et 96 $\mathrm{m}^{2}$ (Larapinta) pour un maximum de 8 personnes. Même la maison la plus petite a trois chambres à coucher, un séjour, une salle à manger, une salle de bain et une cuisine. Il est très probable qu'un argument décisif pour les acheteurs (autre qu'habiter dans des maisons individuelles) est l'existence d'un «Club de Campo " (traduction espagnole de country club) où les résidents pourront louer des salons pour organiser des fêtes privées. À cela s'ajoutent des projets de piscine, de terrains de tennis, de football et des aires de jeux.

Le projet de Valle Grande (figure 3), au contraire de l'Hacienda Urbana Larapinta, reste plus proche de la ville de Santiago, avec une bonne desserte par l'autoroute 5 Norte, qui mène directement au centre-ville en seulement quinze minutes (sauf aux heures de pointe). Le parc industriel homonyme est localisé dans le prolongement de Valle Grande et, contrairement au projet de Larapinta, l'offre résidentielle vise à attirer des acheteurs par l'implantation à venir d'équipements et d'infrastructures tels que des établissements sportifs, d'éducation et des universités.

Figure 3 : Les logements dans les AUDP de Larapinta et de Valle Grande.

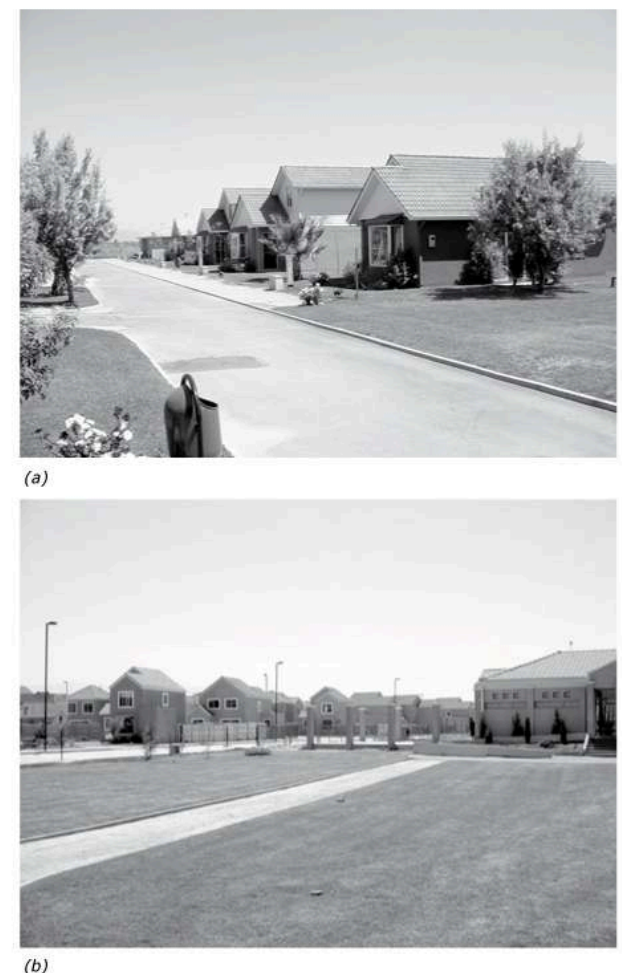

Photographies : (a) Larapinta (Rodrigo Hidalgo, janvier 2005) - (b) Valle Grande (Rodrigo Hidalgo, janvier 2005) 
s les deux ensembles résidentiels, les ventes ont commencé durant l'année 2004 avec respectivement 150 et 550 logements vendus. Pour l'étape finale à Valle Grande on s'attend à vendre de 12000 à 15000 unités, lesquelles hébergeraient environ 60000 habitants. A Larapinta on estime pouvoir vendre 8500 unités pour environ 50000 habitants (tableau 1).

La figure 4 montre les mall's et autres centres commerciaux prévus ou déjà en construction, ainsi que les ZODUC et les AUDP situées au nord de l'AMS. Les autoroutes urbaines, les mall's et les "villes clôturées " sont une expression claire du nouveau modèle d'organisation urbaine qui tend à se diffuser dans une aire considérable de la périphérie de l'AMS et implique une modification notable du mode de vie traditionnel.

Figure 4 : Mégaprojets des quartiers fermés, autoroutes et mall's : nouveaux points de repère de l'organisation urbaine dans le nord de l'AMS, Province de Chacabuco, Santiago du Chili.

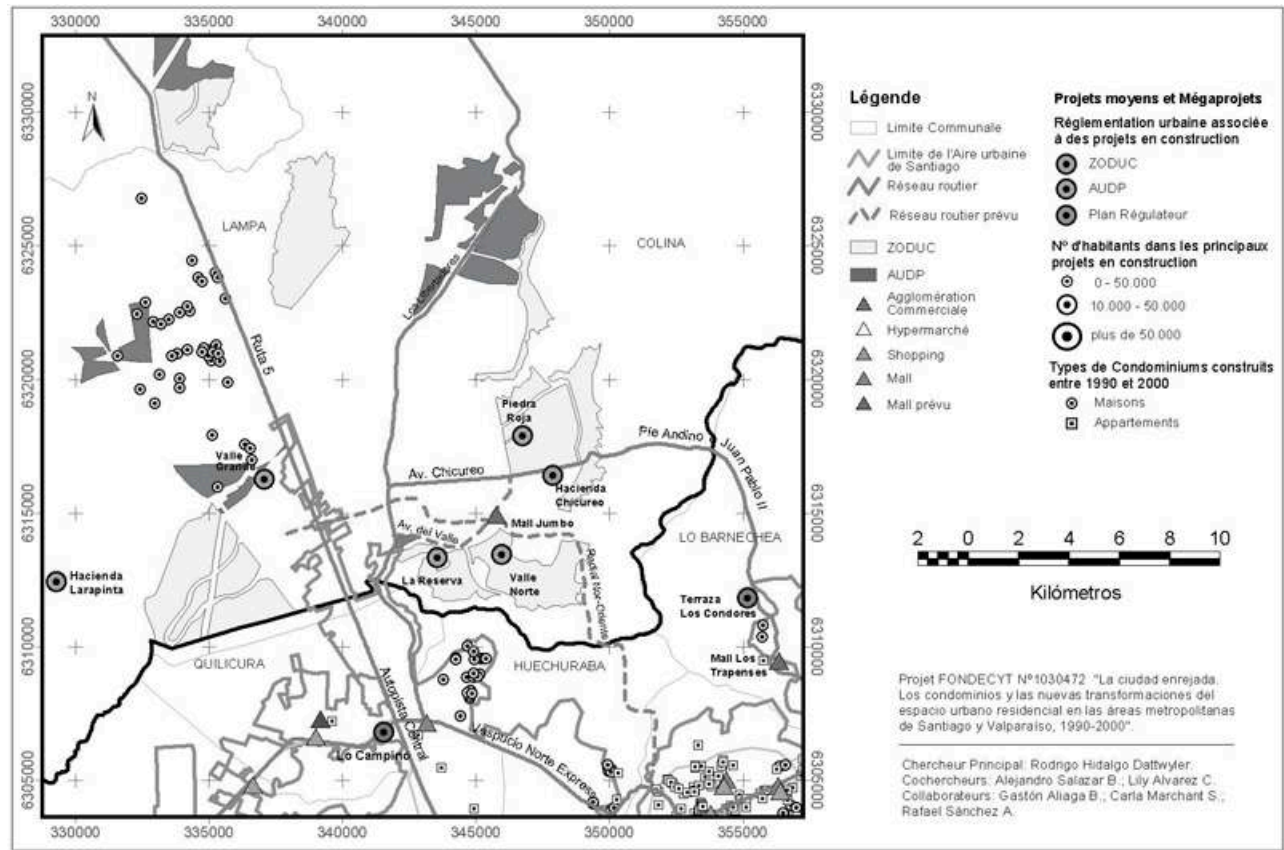

\section{Post-suburbia ou post-urbia ? : les nouvelles modalités de la vie urbaine dans les mégaprojets}

L'une des caractéristiques les plus remarquables du développement urbain en Amérique latine est la fragmentation de la ville en de nombreuses petites unités spécialisées, en relation avec leurs fonctions. L'ancienne bipolarité avec la division classique entre une ville pour les "riches » et une autre pour les "pauvres», en vigueur jusqu'aux années 1970, a été dépassée. Des isolats pour les "gens fortunés » (comme les quartiers fermés) s'étalent partout dans l'aire urbaine. Les « temples de la consommation ", c'est à dire les grands centres commerciaux et les grands magasins, ne sont désormais plus un privilège des quartiers aisés, mais ils ont essaimé partout dans la ville. 

aussi dans les nouveaux schémas de localisation des prestations de services et des complexes industriels. Ainsi, les bâtiments de bureaux ne sont plus construits uniquement dans les quartiers aisés traditionnels; en effet, l'ancien noyau de la ville et les espaces avoisinants, encore en déclin durant les années 1980, sont marqués à nouveau par de forts élans de croissance verticale. Finalement, le secteur industriel tend à se regrouper de plus en plus en parcs d'entreprises petites ou moyennes et à s'éloigner des emplacements manufacturiers traditionnels, auparavant localisés le long des principales routes d'accès et des voies ferrées des grandes villes. Tout cela peut être observé avec un certain détail à Santiago du Chili. Quelques chercheurs ont essayé de définir et de comprendre ce processus de transformation métropolitaine, parmi eux, Carlos de Mattos a proposé la thèse de "l'hybridation " pour le cas des quartiers fermés (de Mattos, 2003). Pour notre part, nous posons la problématique du concept de culture urbaine en Amérique latine, et la question de sa résistance par la fragmentation et le développement «hybride » dans les périphéries métropolitaines. Les « villes clôturées » émergentes, ne constituent-elles pas des modes de vie post-suburbain (Borsdorf, 2004) ou encore post-urbain? Ces concepts urbains implantés en Amérique latine jusqu'à saturation, ne sont-ils que des manifestations de la mentalité agricole américaine infiltrée à travers les canaux de la mondialisation dans une culture complètement différente?

41 En pondérant les critères définis par H. Bobek (1927) sur ce qui définit l'urbain, les mégaprojets réunissent seulement les conditions de densité et de dimension. À l'instar des ghettos, ils sont seulement monofonctionnels; leurs habitants peuvent utiliser uniquement les équipements et les services construits pour chaque projet. Les activités $\mathrm{du}$ secteur secondaire restent exclues et la «vie urbaine » n'est pas entièrement présente, notamment parce qu'elle pourrait être la source de problèmes. D'autres sondages ont démontré que les infrastructures communautaires (surtout celles des quartiers fermés pour les groupes plus aisés de la société) ne sont pratiquement pas utilisées, peut être parce que leur esprit élitiste est incompatible avec ce qui est collectif. Ceci se rapproche énormément du mode de vie aristocratique-rurale, très éloigné, sans aucun doute, de l'urbain. En effet, la publicité pour les «villes clôturées " fait écho à l'image idyllique de la vie paisible à la campagne, ici transformée en réalité. Ce message principal est également répercuté auprès des couches moyennes et moyennes inférieures de la société, à travers les prospectus faisant la promotion des complexes résidentiels où en réalité les habitants vivront plutôt entassés.

42 Cependant, le plus discutable dans ces grands complexes où demeureront environ 50000 personnes, est l'absence de contact ordinaire entre les différents groupes sociaux qui constituent la diversité sociale des villes. La sélection opérée par le prix de vente des logements dans les immeubles, même ceux qui sont les moins chers, empêche toute sorte de contact avec d'autres couches sociales, ce qui nous amène à attendre pour l'avenir une mobilité plutôt horizontale que verticale, comme aux États-Unis. Cela signifie que la promotion d'un individu dans l'échelle sociale le conduira à la recherche d'un nouveau quartier résidentiel, pour y déménager, au lieu de rester dans son ancien quartier et d'investir dans sa propriété.

43

Rien de plus éloigné de l'urbanité au sens classique du terme, que ce processus. Si l'on analyse en profondeur les "villes clôturées ", celles-ci se révèlent comme l'antithèse de la ville, car l'individualisme est mis en avant au détriment du civisme, l'idylle de la paix 
rurale au détriment de la vie collective, ce qui est excluant par rapport à ce qui est incluant. Les nouvelles tendances se manifestent comme des modèles contraires au concept traditionnel de ville, puisqu'elles invalident ses fonctions principales telles que l'accessibilité, l'utilisation publique des réseaux de transport, de commerce, des établissements éducationnels et d'autres prestations de services, ainsi que la présence d'individus socialement hétérogènes. Il faudrait encore se poser la question de la durabilité de ce mode de vie et sur le fait que les « riches " puissent subsister sans les "pauvres ", leur bien-être étant finalement très dépendant des services que ceux-ci leur rendent.

\section{BIBLIOGRAPHIE}

вовек Н. (1927). - « Grundfragen der Stadtgeographie », Geographischer Anzeiger, 28, p. 213-224.

BORSDORF A. (2000). - «Condominios in Santiago de Chile als Beispiele sozialräumlicher Segregationstendenzen von Ober- und Mittelschicht in lateinamerikanischen Städten »,Peripherie, Zeitschrift für Politik und Ökonomie in der Dritten Welt, 20, 80, p. 25-40.

BORSDORF A. (2002). - « Vor verschlossenen Türen - Wie neu sind Tore und Mauern in lateinamerikanischen Städten? ", Geographica Helvetica, 57, 4, p. 278-289.

BORSDORF A., BÄHR J., JANOSCHKA M. (2002). - « Die Dynamik stadtstrukturellen Wandels im Modell der lateinamerikanischen Stadt », Geographica Helvetica, 57, 4, p. 300-310.

BORSDORF A. (2004a). - « Commercial areas in the outskirts of European cities ». In : BORSDORF A. \& ZEMBRI P., European cities. Insight on outskirts, Paris, METL/PUCA., p. 129-148.

BORSDORF A. (2004b). - « On the way of post-suburbia ? Changing structures in the outskirts of European cities ». In : BORSDORF A. \& ZEMBRI P., European cities. Insight on outskirts, Paris, METL/ PUCA., p. 7-30.

BORSDORF A. \& HIDALGO R. (2004a). - « Formas tempranas de exclusión residencial y el modelo de la ciudad cerrada en América Latina. El caso de Santiago », Revista de Geografía Norte Grande, 32, p. 21-37.

BORSDORF A. \& HIDALGO R. (2004b). - « Vom barrio cerrado zur ciudad vallada. Neue Dimensionen der sozialen und funktionalen Exklusion in Santiago de Chile ", Mitteilungen der Österreichischen Geographischen Gesellschaft, 146, p. 111-124.

BORSDORF A. \& HIDALGO R. (2005). - « Los megadiseños residenciales vallados en las periferias de las metrópolis latinoamericanas y el advenimiento de un nuevo concepto de ciudad. Alcances en base al caso de Santiago de Chile ». In : VII COLOQUIO INTERNACIONAL DE GEOCRÍTICA. Los agentes urbanos y las políticas sobre la ciudad. Santiago, Instituto de Geografía - Pontificia Universidad Católica de Chile de Chile y Departamento de Geografía Humana Universidad de Barcelona, 24-27 de mayo 2005. 
CÁCERES G. \& SABATINI F. (2004). - Barrios cerrados en Santiago de Chile. Entre la exclusión y la integración residencial, Santiago, Lincon Institute e Instituto de Geografía, Pontificia Universidad Católica de Chile.

CASTRO c. (2005). - « Impacto de la dispersión urbana de la ciudad de Santiago en la calidad del suelo en la periferia norte : Colina y Lampa ». In : VII COLOQUIO INTERNACIONAL DE GEOCRÍTICA. Los agentes urbanos y las políticas sobre la ciudad. Santiago, Instituto de Geografía-Pontificia Universidad Católica de Chile de Chile y Departamento de Geografía Humana, Universidad de Barcelona, 24-27 de mayo 2005.

COY M., PÖHLER M. (2002). - « Gated communities in Latin American megacities : case studies in Brazil and Argentina ", Environment and Planning B : Planning and Design, 29, p. 355-370.

DE MATTEIS G. (1998). - « Suburbanización y periurbanización. Ciudades anglosajonas y ciudades latinas ». In : MONCLÚS, F., La ciudad dispersa, Barcelona, Centro de Cultura Contemporània de Barcelona, p. 17-34.

DE MATTOS C. (2003). - « Globalización y transformación metropolitana en el caso de Santiago ». In : ARENAS F ; HIDALGO R. \& COLL J., Los nuevos modos de gestión de la metropolización, Santiago, Serie Geolibros, p. 27-55.

DOLLfuS O. (1997). - La mondialisation, Paris, La Bibliothèque du Citoyen, Presses de la FNSP.

GLASZE G.; WEBSTER C. \& FRANTZ K. (ed) (2005). - Private Neighbourhoods. Global and local perspectives, London, Routledge.

HARVEY D. (1990). - The condition of postmodernity : an enquiry into the origins of cultural change, Cambridge, Blackwell.

HIDALGO R. (2004). - « De los pequeños condominios a la ciudad vallada : las urbanizaciones cerradas y la nueva geografía social en Santiago de Chile (1990-2000) », EURE, 2004, Tome 30, 91, p. 29-52.

HIDALGO R., BORSDORF A. (2005). - « Barrios cerrados y fragmentación urbana en América Latina : estudio de las transformaciones socioespaciales en Santiago de Chile (1990-2000) ». In : HIDALGO R.; TRUMPER R. \& BORSDORF A.,Transformaciones urbanas y procesos territoriales. Lecturas del nuevo dibujo de la ciudad latinoamericana, Santiago, Serie GEOlibros, Academia de Ciencias Austriaca y Okanagan University College, p. 105-122.

HIDALGO R., SALAZAR A., LAZCANO R., ROA F. (2003). - « Periurbanización y condominios en el área metropolitana de Santiago de Chile. El caso de Pirque y Calera de Tango », Revista Geográfica de Chile Terra Australis, 48, p. 117-138.

INSTITUTO NACIONAL DE ESTADÍsTiCAS (2002). - Glosario Censo, Santiago, Ine.

JANOSCHKA M. (2002). - « “Stadt der Inseln". Buenos Aires : Abschottung und Fragmentierung als Kennzeichen eines neuen Stadtmodells », RaumPlanung, 101, p. 65-70.

KANITSCHEIDER S. (2002). - « Condominios und Fraccionamientos Cerrados in Mexiko-Stadt. Sozialräumliche Segregation am Beispiel abgesperrter Wohnviertel », Geographica Helvetica, 57, 4, p. 253-263.

KOHLER P. (2002). - « Geschlossene Wohnkomplexe in Quito. Naturraum und rechtliche Rahmenbedingungen als Einflußgrößen für Verbreitung und Typisierung », Geographica Helvetica, 57, 4, p. 278-289. 
LACARRIEU M., THUILLIER G. (2004). - « A utopia of order and enclosure : « private districts » and « countries » in Buenos Aires ». L'Espace géographique, 2/04, p. 149-164.

MARCUSE P, KEMPEN R. (eds.). (2000). - Globalizing Cities : A New Spatial Order ?, London and Cambridge, Blackwell Publishers.

MERTINS G. (2003). - « Jüngere sozialräumlich-strukturelle Transformation in den Metropolen und Megastädten Lateinamerikas », Petermanns Geographische Mitteilungen, 147, p. 46-55.

MEYER K., BÄHR J. (2001). - « Condominios en Greater Santiago de Chile and their Impact on de Urban Structure », Die Erde, 132, p. 293-321.

MINISTERIO DE AGRICULTURA. (1980). - Decreto Ley 3516, establece subdivisión de Predios Rústicos, Santiago, Ministerio de Agricultura.

MONCLUS F. (1998). - « Suburbanización y nuevas periferias. Perspectivas geográfico-

urbanísticas ». In : MONCLUS F., La ciudad Dispersa, Barcelona, Centro de Cultura Contemporánea de Barcelona.

PARNREITER C., FISHER K., JÄGER J. (2005). - « La metrópolis latinoamericana como nodo central en las cadenas productivas globales. Transformaciones socioeconómicas y desarrollo urbano ». In : HIDALGO R. ; TRUMPER R. \& BORSDORF A., Transformaciones urbanas y procesos territoriales. Lecturas del nuevo dibujo de la ciudad latinoamericana, Santiago, Serie GEOlibros, Academia de Ciencias Austriaca y Okanagan University College, p. 45-56.

ORTIZ J., MORALES S. (2002). - « Impacto socioespacial de las migraciones intraurbanas en entidades de centro y de nuevas periferias del Gran Santiago ", EURE,.85, p. 171-185.

PODUJE I., YAÑEZ G. (2000). - « Planificando la ciudad virtual : megaproyectos urbanos estatales y privados ». In : Seminario Internacional Las regiones metropolitanas del Mercosur y México : entre la competitividad y la complementariedad, Buenos Aires, Programa de Investigación Internacional Grandes Regiones Metropolitanas del Mercosur y México.

PREVOT M. (2002). - « Buenos Aires en los años ‘90 : Metropolización y desigualdades ». EURE, 85, pp 31-50.

PREVOT M. (2000). - « Segregación, fragmentación, secesión. Hacia una nueva geografía social en la Aglomeración de Buenos Aires », Revista Economía, Sociedad y Territorio, Tome II, 7, p. 405-431.

SASSEN S. (1994). - Cities in a World Economy, London, New Delhi.

THUILLIER G. (2000). - « Les quartiers enclos à Buenos Aires : Quand la ville devient country ». Cahiers des amériques latines, 35, p. 41-56.

\section{NOTES}

1. Projets Fondecyt 1030472,7050123 et 7040113.

2. Note de l'auteur.

3. L'AMS a été définie à partir de l'aire urbanisée continue, laquelle, selon l'information satellitaire de novembre 2001, est composée de 39 communes: Santiago, Independencia, Conchalí, Huechuraba, Recoleta, Providencia, Vitacura, Lo Barnechea, Las Condes, Ñuñoa, La Reina, Macul, Peñalolén, La Florida, San Joaquín, La Granja, La Pintana, San Ramón, San Miguel, La Cisterna, El Bosque, Pedro Aguirre Cerda, Lo Espejo, Estación Central, Cerrillos, Maipú, Quinta Normal, Lo Prado, Pudahuel, Cerro Navia, Renca, Quilicura, Colina, Lampa, Puente Alto, San Bernardo, Padre Hurtado, Pirque y Calera de Tango. 
4. Outil d'aménagement territorial ressemblant aux Schèmas Directeurs d'Aménagement Urbain (SDAU) français.

\section{RÉSUMÉS}

À partir de l'étude des nouveaux grands projets résidentiels clôturés de la périphérie nord de l'aire métropolitaine de Santiago du Chili, on analyse la manière dont ces interventions modèlent une nouvelle image de l'expansion urbaine, organisée sur la base du tracé des autoroutes urbaines et des grandes surfaces ou centres commerciaux (malls). En considérant ces éléments, on établit quelques hypothèses qui montrent que cette nouvelle configuration spatiale de la ville conduit à un concept de ville éloigné de la diversité sociale et fonctionnelle pourtant caractéristique de la formation du complexe urbain.

On the basis of study of recent studies regarding the construction of «fenced cities " on the northern area of the Metropolitan Region of Santiago, Chile, this paper analyzes the ways in which these interventions are redefining the urban expansion process, which is now being organized around new urban highways and large commercial centers such as malls. Taking into account these elements, several hypotheses are proposed, pointing out that the resulting urban configuration is driving the city away from the social and functional diversity characteristic of urban settlements.

Auf der Grundlage neuerer Studien zur Ausbildung von «eingezäunten Städten », d.h. von Wohneinheiten von 50.000 und mehr Einwohnern, im Norden der Metropolitanregion von Santiago de Chile, untersucht der Artikel die Art und Weise, wie solche Entwicklungen den Stadtentwicklungsprozess re-definieren. Die neuen Strukturen sind durch nur für die Bewohner zugängliche Privatautobahnen, Einkaufszentren und Bildungseinrichtungen gekennzeichnet. Es stellt sich die Frage, ob solche post-suburbanen Entwicklungen die Idee der Stadt nicht konterkarieren, indem viele Charakteristiken von Städten - soziale Durchmischung, Öffentlichkeit, Funktionsvielfalt, Zentralität - nicht mehr erfüllt werden.

\section{INDEX}

Mots-clés : morphologie urbaine, périphérie métropolitaine, résidence clôturée

Keywords : gated communities, metropolitan periphery, urban morphology

Schlüsselwörter : eingezäunte Siedlung, metropolitane Peripherie, städtische Morphologie

\section{AUTEUR}

RODRIGO HIDALGO D

Institut de Géographie, Pontifice Université Catholique du Chili 